\title{
Preoperative Topical Antimicrobial Decolonization in Head and Neck Surgery
}

\author{
Andrew G. Shuman, MD; Emily K. Shuman, MD; Samantha J. Hauff, MD; Laura L. Fernandes, MS; \\ Emily Light, MS; Carol E. Chenoweth, MD; Carol R. Bradford, MD
}

\begin{abstract}
Objectives/Hypothesis: Surgical site infections (SSIs) are an important cause of morbidity and mortality after head and neck surgery. Our primary objective was to determine the efficacy of preoperative topical antimicrobial decolonization before head and neck surgery.

Study Design: Prospective, randomized controlled trial.

Methods: This study was conducted among 84 patients presenting for head and neck surgery requiring admission to an academic medical center. Preoperative cultures were performed to identify Staphylococcus aureus carriers. Patients were randomized to preoperative topical antimicrobial decolonization with a 5-day regimen of chlorhexidine skin rinses and intranasal mupirocin coupled with standard perioperative systemic antimicrobial prophylaxis, versus standard prophylaxis alone. The main outcome was the incidence of SSIs.

Results: Despite a trend suggesting a decrease in SSIs with perioperative topical antimicrobial decolonization (24\% vs. $10 \%$ ), there was no significant difference (odds ratio, $0.34 ; 95 \%$ confidence interval, $0.10-1.18 ; P=.079$ ). Patients with a higher American Society of Anesthesiologists score (3 vs. $1 ; P=.02)$, with more operative blood loss $(P=.05)$, and who required operative takeback $(P=.04)$ had a higher rate of SSIs; there was a trend suggesting a higher rate of SSIs among patients undergoing clean-contaminated surgery compared to clean cases $(P=.08)$ and among those having received prior radiation $(P=.07)$ or chemotherapy $(P=.06)$.

Conclusions: Preoperative antimicrobial decolonization did not significantly decrease the incidence of SSIs after head and neck surgery, but might be considered for high-risk groups despite the lack of conclusive evidence confirming efficacy. Risk factors for SSIs after head and neck surgery are identified for the first time in a prospective study.

Key Words: Surgical site infection, antimicrobial decolonization, head and neck surgery.

Level of Evidence: $1 \mathrm{~b}$
\end{abstract}

Laryngoscope, 122:2454-2460, 2012

\section{INTRODUCTION}

Surgical site infections (SSIs) are an important cause of morbidity and mortality after head and neck surgery, causing worsened prognosis, prolonged hospitalization, and poor cosmetic results. Incidence ranges from $8 \%$ to $45 \%$, even with standard antimicrobial prophylaxis., ${ }^{1,2}$ Several studies have demonstrated the

From the Departments of Otolaryngology-Head and Neck Surgery (A.G.S., S.J.H., C.R.B.) and Internal Medicine, Division of Infectious Disease, University of Michigan Medical School (E.K.S., C.E.C.); Department of Biostatistics, University of Michigan School of Public Health (L.L.F.), University of Michigan Comprehensive Cancer Center, Biostatistics Core (E.L.): and Department of Infection Control and Epidemiology, University of Michigan Medical School (c.E.c.), Ann Arbor, Michigan, U.S.A. $11,2012$.

Editor's Note: This Manuscript was accepted for publication May

Presented at the Triological Society Combined Sections Meeting, San Diego, California, U.S.A., April 18-22, 2012.

One of the study medications was donated by Hibiclens, Mölnlycke Health Care, Inc. All funding was obtained via internal funding sources. Mölnlycke Health Care did not participate in or approve of the design, analysis, or publication of this study.

The authors have no other funding, financial relationships, or conflicts of interest to disclose.

Send correspondence to Andrew G. Shuman, MD, Department of Otolaryngology-Head and Neck Surgery, University of Michigan Hospitals, 1904 Taubman Center, Ann Arbor, MI 48109.

E-mail: shumana@umich.edu

DOI: 10.1002/lary.23487 importance of Staphylococcus aureus as an important pathogen in SSIs after head and neck surgery. ${ }^{1,2}$

Most SSIs after head and neck surgery are caused by contamination of the surgical wound with endogenous flora present on the skin or in the aerodigestive tract. ${ }^{3}$ Topical antimicrobial decolonization is one strategy used to mitigate the risk of SSIs. Mupirocin is a topical antimicrobial that is active against most staphylococci and streptococci but less active against Gram-negative bacilli and anaerobes. ${ }^{4}$ Chlorhexidine is an agent with broad antiseptic activity that is utilized in multiple clinical settings. ${ }^{5}$ A recent randomized controlled trial suggested that preoperative decolonization of $S$. aureus carriers utilizing intranasal mupirocin effectively decreases the risk for SSIs, a finding that has been corroborated by large meta-analyses. ${ }^{6-8}$ Data regarding the effectiveness of preoperative decolonization with chlorhexidine have been less convincing. ${ }^{9}$ The majority of data concerning the effectiveness of decolonization in preventing SSIs apply to orthopedic and cardiac surgery; this has not been addressed among head and neck surgical patients. ${ }^{10}$

We sought to address several important issues related to the role of topical antimicrobial decolonization prior to head and neck surgery. Our first aim was to determine the efficacy of preoperative topical decolonization with mupirocin and chlorhexidine (regardless of 
colonization status) on the rate of SSIs. Our second aim was to determine the proportion of patients colonized with methicillin-sensitive $S$. aureus (MSSA) or methicillin-resistant $S$. aureus (MRSA) preoperatively by sampling several relevant anatomic sites (anterior nares, throat, and skin of nape of neck). We also sought to determine independent predictors of SSIs in patients undergoing head and neck surgery.

\section{MATERIALS AND METHODS}

\section{Study Design and Enrollment}

This study was conducted at a large Midwestern academic medical center. The protocol was reviewed and approved by the University of Michigan Institutional Review Board. Subjects were recruited in the preoperative otolaryngology clinic and consisted of all patients scheduled to undergo elective head and neck surgery requiring admission to the hospital postoperatively. Written informed consent was obtained prior to enrollment in the study. Exclusion criteria included patients undergoing ambulatory procedures, known hypersensitivity to either mupirocin or chlorhexidine, immunosuppression due to underlying illness or medications, and a documented preoperative infection involving the surgical field.

Once patients were enrolled, sterile swab specimens (BactiSwab; Starplex Scientific, London, Ontario, Canada) were obtained from the anterior nares, oropharynx, and the skin on the nape of the neck. Specimens were sent to the microbiology laboratory for identification of $S$. aureus (both MSSA and MRSA) using standard culture-based techniques.

\section{Intervention}

Patients were randomized into two groups based upon the last digit of their medical record numbers. Patients in the experimental group were assigned to undergo preoperative topical decolonization with mupirocin $2 \%$ ointment applied intranasally each day for the 5 days leading up to surgery and $2 \%$ chlorhexidine gluconate solution (Hibiclens; Mölnlycke Health Care, Norcross, GA) used daily during routine bathing for the same time interval. Patients in the control group did not undergo topical decolonization. In the event of any hypersensitivity or adverse reaction to these medications, the medication was discontinued, and the patient was disqualified from the study.

Patients who did not culture positive for $S$. aureus, as well as those found to be colonized with MSSA, were given routine perioperative antimicrobial prophylaxis according to our standard institutional protocol and in accordance with recommendations from the Centers for Disease Control and Prevention (CDC). ${ }^{11,12}$ This consisted of intravenous ampicillin/ sulbactam or clindamycin for clean-contaminated cases and cefazolin for clean cases given for no more than 23 hours postoperatively, with the first dose given within 1 hour prior to surgical incision; patients were redosed intraoperatively in accordance with the standard pharmacologic protocol. Patients colonized with MRSA received perioperative antimicrobial prophylaxis directed against MRSA (i.e., intravenous vancomycin) in addition to routine perioperative antimicrobial prophylaxis, as indicated based upon the surgical procedure.

\section{Definition of Primary Outcome}

Patients were followed for 30 days postoperatively to monitor for the development of SSIs, which was defined according to the surveillance definition from the CDC and National Healthcare Safety Network (NHSN). ${ }^{13}$ SSIs were identified by review of medical records for all patients enrolled in the study and were classified as either major (requiring hospital readmission, additional surgery, or prolonged hospitalization, or resulting in permanent morbidity/mortality) or minor (all others). Fistulae alone were not categorized as SSIs in the absence of CDC/NHSN criteria for SSIs. Wound cultures were obtained when appropriate.

\section{Identification of SSI Risk Factors and Potential Confounders}

Patient gender, age, and diabetic status were determined at the time of enrollment. Tobacco use was stratified among current smokers (any tobacco within past month), ex-smokers (any tobacco prior to past month), and never smokers. Any prior history of radiation therapy or chemotherapy targeted against head and neck neoplasms was also recorded. Swab results were interpreted and categorized into whether patients were MRSA or MSSA carriers and, if so, from which anatomic sites.

Surgical wounds were categorized as clean, clean-contaminated, or dirty based upon established definitions. ${ }^{14}$ The American Society of Anesthesiologists (ASA) Physical Status classification system was employed to describe patients' overall perioperative health status. ${ }^{15,16}$ Surgical sites were described, as was the need for additional unplanned surgical procedures within 30 days of the initial operation (deemed operative takebacks). Estimated blood loss for the surgical procedures and operative case length (from incision to surgical dressing) were extracted from an electronic, prospectively collected perioperative database. The study protocol specified that perioperative antimicrobials were discontinued within 1 day of surgery unless extenuating circumstances existed; violations of this protocol were recorded along with the reasons for these events.

\section{Statistical Analysis}

A power calculation was performed during study design. The primary endpoint was the presence or absence of SSIs at 30 days. Sample size was determined by calculating the number of subjects (36 patients in each arm) required to detect a $25 \%$ difference in the rate of SSIs assuming a baseline infection rate of $30 \%$ (based on unpublished institutional epidemiologic data and review of the scientific literature ${ }^{1}$ ). We chose a $25 \%$ difference because it seemed reasonable from a clinical standpoint, and because it was feasible given the overall study design within a single institution's surgical practice.

Descriptive statistics were calculated; frequencies and percentages were presented for categorical variables, and means and standard deviations were presented for quantitative measures. The balance of potential confounders across control versus experimental groups was assessed using a $\chi^{2}$ test of association for categorical variables and $t$ tests for continuous variables. Logistic regression was performed on the incidence of SSIs, the primary outcome, as a binary variable with group assignment in the model to test the main hypothesis regarding the benefit of preoperative topical antimicrobial decolonization before head and neck surgery. The odds ratios (ORs) for various SSI risk factors were calculated using logistic regression after adjusting for group assignment in the model. The OR estimate for group assignment was monitored in all models to determine whether any risk factors affected the estimates of group assignment effect. Statistical analysis was performed using SAS software (Cary, NC).

\section{RESULTS}

\section{Enrollment and Protocol Adherence}

A total of 88 patients were consented, enrolled, and randomized (Fig. 1). No patients in the experimental 


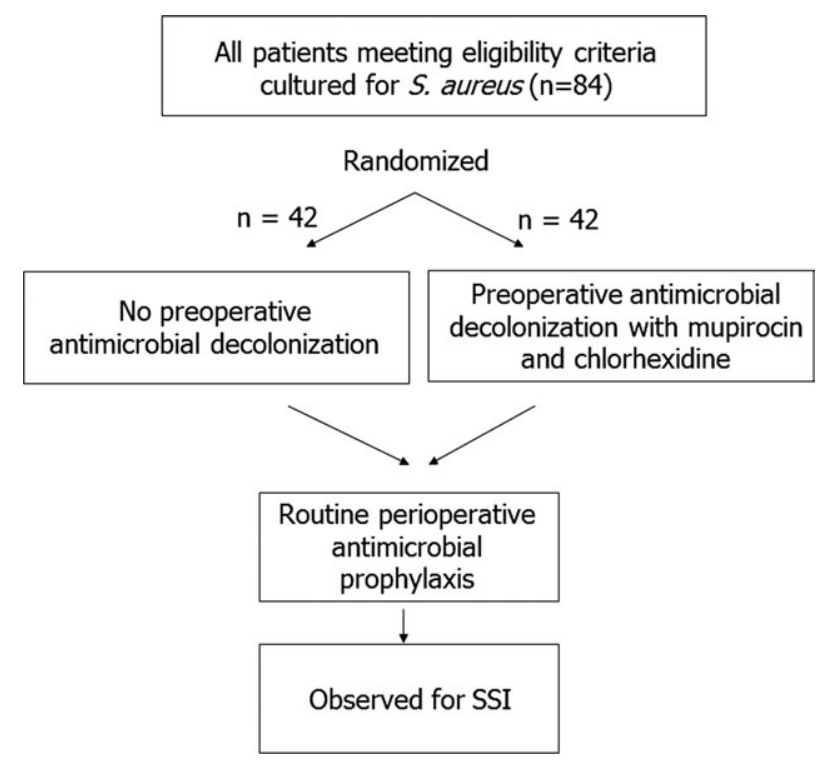

Fig. 1. Study design algorithm. SSI = surgical site infection.

group reported any complications with the decolonization regimen. Two patients, each from the control and experimental groups, were excluded for nonadherence to the study protocol. Two subjects (one in each arm) died within 30 days of surgery of non-SSI-related causes, and were kept in the analysis with follow-up for SSIs until the date of their death. Thus, a total of 84 patients (42 in each arm) were utilized for the data analysis.

A total of 18 patients were treated with more than the recommended 23-hour course of perioperative antibiotics; seven were in the control group, and 11 were in the experimental group $(P=.29)$. The rationale for extending antibiotics was attending surgeon preference/ specification $(\mathrm{n}=11)$, urinary tract infection $(\mathrm{n}=3)$, and unclear reasons $(\mathrm{n}=4)$.

Statistical evaluation of balance across the two groups revealed that the patients in the two arms of the study were well matched. There were no significant differences among the control and experimental groups with regard to any demographic, clinical, or perioperative characteristics at the $P=.10$ level or below (Tables I and II).

\section{Primary Outcome}

There was a $24 \%$ incidence $(\mathrm{n}=10)$ of SSIs in the control group versus a $10 \%$ incidence $(n=4)$ of SSIs in the experimental group (Fig. 2). The number needed to treat (calculated number of patients treated with the experimental protocol to prevent one SSI) was seven. Despite the trend suggesting a decrease in SSIs with the institution of preoperative topical antimicrobial decolonization, there was no statistically significant difference in the primary outcome between these two groups (OR, 0.338; 95\% confidence interval [CI], 0.096-1.177; $P=.079$ ).

Of the 10 infections in the control group, four were major, and six were minor. The major infections included two complex neck wounds with fistulae, a case of necrot- izing soft tissue infection of the surgical site, and a deep neck space infection requiring readmission. Two of the major infections were amenable for culture; one culture grew both coagulase-negative Staphylococcus and Eikenella; the other grew MRSA. Of the four infections in the experimental group, all were categorized as minor; only one of these (a superficial wound infection with dehiscence) was able to be cultured, and the culture grew MRSA.

Subgroup analyses were conducted to determine if the primary outcome was significantly different among subsets of patients undergoing decolonization compared to the control group. There was no specific subset of patients for whom the study intervention significantly decreased the risk of SSIs. These data are presented in Table III.

\section{S. aureus Carrier Status}

A total of $26(31 \%)$ of all patients were S. aureus carriers. Six $(7 \%)$ patients carried MRSA, and $20(24 \%)$ carried MSSA. MRSA was cultured from the nares/neck skin in three patients, the oropharynx in one patient, and all sites in two patients. MSSA was cultured from the nares/neck skin in 13 patients and all sites in seven patients. Of the MRSA carriers, there were two minor

TABLE I.

Preoperative Sample Characteristics of Study Population.

\begin{tabular}{|c|c|c|c|c|}
\hline Characteristic & $\begin{array}{l}\text { Control } \\
\text { Group }\end{array}$ & $\begin{array}{l}\text { Experimental } \\
\text { Group }\end{array}$ & Total & $P$ \\
\hline No. & $42(50 \%)$ & $42(50 \%)$ & 84 & \\
\hline Gender & & & & .66 \\
\hline Male & $25(60 \%)$ & $23(55 \%)$ & 48 (57\%) & \\
\hline Female & $17(40 \%)$ & $19(45 \%)$ & $36(43 \%)$ & \\
\hline Patient age, yr & & & & .32 \\
\hline Mean & 59.76 & 56.52 & 58.14 & \\
\hline Median & 61 & 57 & 57.5 & \\
\hline Tobacco use & & & & .13 \\
\hline Current & $10(24 \%)$ & 8 (19\%) & $18(21 \%)$ & \\
\hline Never & $13(31 \%)$ & $22(52 \%)$ & 35 (42\%) & \\
\hline Former & $19(45 \%)$ & $12(29 \%)$ & $31(37 \%)$ & \\
\hline Prior chemotherapy & & & & .50 \\
\hline Yes & $6(14 \%)$ & $4(10 \%)$ & $10(12 \%)$ & \\
\hline No & $36(86 \%)$ & 38 (90\%) & 74 (88\%) & \\
\hline Prior surgery & & & & .66 \\
\hline Yes & $17(40 \%)$ & $19(45 \%)$ & $36(43 \%)$ & \\
\hline No & $25(60 \%)$ & $23(55 \%)$ & $48(57 \%)$ & \\
\hline Prior radiation & & & & .43 \\
\hline Yes & $11(26 \%)$ & 8 (19\%) & $19(23 \%)$ & \\
\hline No & $31(74 \%)$ & $34(81 \%)$ & $65(77 \%)$ & \\
\hline Diabetic & & & & 1.00 \\
\hline Yes & $5(12 \%)$ & $5(12 \%)$ & $10(12 \%)$ & \\
\hline No & $37(88 \%)$ & 37 (88\%) & 74 (88\%) & \\
\hline S. aureus carrier & & & & .35 \\
\hline Yes & $11(26 \%)$ & $15(36 \%)$ & $26(31 \%)$ & \\
\hline No & 31 (74\%) & 27 (64\%) & 58 (69\%) & \\
\hline
\end{tabular}


TABLE II.

Perioperative Sample Characteristics of Study Population.

\begin{tabular}{|c|c|c|c|c|}
\hline Characteristic & $\begin{array}{l}\text { Control } \\
\text { Group }\end{array}$ & $\begin{array}{l}\text { Experimental } \\
\text { Group }\end{array}$ & Total & $P$ \\
\hline No. & $42(50 \%)$ & $42(50 \%)$ & 84 & \\
\hline \multicolumn{4}{|l|}{ Surgical wound class } & .57 \\
\hline Clean & $21(50 \%)$ & $20(48 \%)$ & $41(49 \%)$ & \\
\hline Clean-contaminated & $20(48 \%)$ & $22(52 \%)$ & 42 (50\%) & \\
\hline Dirty & $1(2 \%)$ & 0 & $1(1 \%)$ & \\
\hline \multicolumn{4}{|l|}{ ASA classification } & .54 \\
\hline 1 & $16(38 \%)$ & $16(38 \%)$ & 32 (38\%) & \\
\hline 2 & $12(29 \%)$ & $16(38 \%)$ & $28(33 \%)$ & \\
\hline 3 & $14(33 \%)$ & $10(24 \%)$ & 24 (29\%) & \\
\hline \multicolumn{4}{|l|}{ Surgical site } & * \\
\hline Integument & $5(12 \%)$ & $3(7 \%)$ & $8(9 \%)$ & \\
\hline Larynx & $7(17 \%)$ & $2(5 \%)$ & $9(11 \%)$ & \\
\hline Neck & $17(40 \%)$ & $21(50 \%)$ & $38(45 \%)$ & \\
\hline Nose/sinus & 0 & $2(5 \%)$ & $2(2 \%)$ & \\
\hline Oral cavity & $9(21 \%)$ & $6(14 \%)$ & $15(18 \%)$ & \\
\hline Pharynx & $1(2 \%)$ & $2(5 \%)$ & $3(4 \%)$ & \\
\hline Skull base & $3(7 \%)$ & $6(14 \%)$ & $9(11 \%)$ & \\
\hline \multicolumn{4}{|l|}{ Antibiotic duration } & .29 \\
\hline$>24$ hours & $7(17 \%)$ & $11(26 \%)$ & $18(21 \%)$ & \\
\hline$<24$ hours & 35 (83\%) & $31(74 \%)$ & 66 (79\%) & \\
\hline \multicolumn{4}{|l|}{ Operative takeback } & .17 \\
\hline Yes & $4(10 \%)$ & $1(2 \%)$ & $5(6 \%)$ & \\
\hline No & 38 (90\%) & $41(98 \%)$ & 79 (94\%) & \\
\hline \multicolumn{4}{|l|}{ Operative EBL } & .28 \\
\hline Mean & 247.14 & 176.30 & 211.72 & \\
\hline Median & 100.00 & 100.00 & 100.00 & \\
\hline \multicolumn{4}{|l|}{ Case length, min } & .69 \\
\hline Mean & 323.16 & 304.19 & 313.67 & \\
\hline Median & 317.00 & 225.50 & 277.50 & \\
\hline
\end{tabular}

*Small numbers in each group obviated statistical analysis.

ASA = American Society of Anesthesiologists Physical Status classification system; EBL = estimated blood loss.

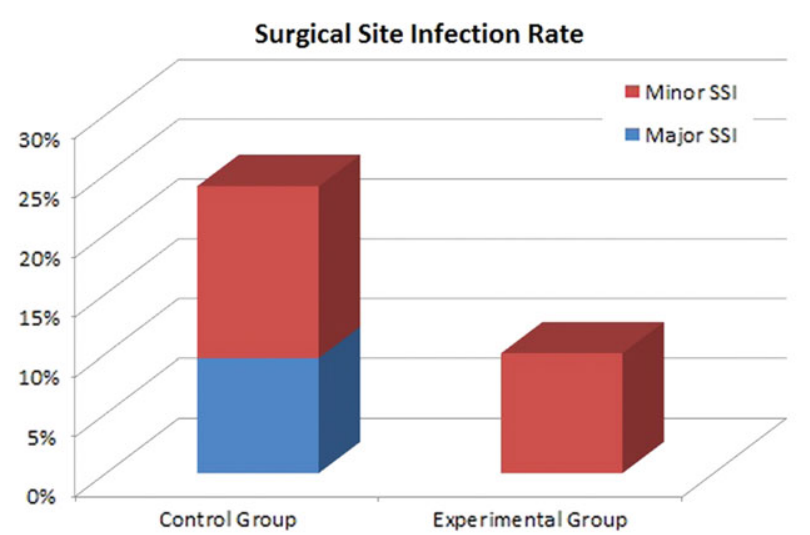

SSI: Surgical Site Infection

* Comparison of SSI incidence between experimental and control group: $\mathrm{OR}=0.338 ; 95 \% \mathrm{Cl} 0.096-1.177 ; \mathrm{p}=0.079$

Fig. 2. Surgical site infection rate. $\mathrm{OR}=$ odds ratio; $\mathrm{Cl}=$ confidence interval. [Color figure can be viewed in the online issue, which is available at wileyonlinelibrary.com.] wound infections, neither of which cultured positive for MRSA. Of the MSSA carriers, there was one minor wound infection, which was not cultured. S. aureus carrier status was not associated with an increased risk for SSIs (OR, 0.64; 95\% CI, 0.15-2.48; $P=.49$ ). The subset of patients colonized with $S$. aureus who underwent decolonization did not derive a significant benefit compared to the subset of patients colonized with $S$. aureus in the control group (OR, 1.54; 95\% CI, 0.12-19.46; $P=$.74).

\section{Risk Factors for SSIs}

Patients with a higher ASA Physical Status classification system score (3 vs. 1; OR, 4.71; 95\% CI, 1.07$20.74 ; P=.02$ ), with more operative blood loss (OR, $1.002 ; 95 \%$ CI, $1.00-1.004 ; P=.05$ ), and who required operative takeback (OR, 7.51; 95\% CI, 1.08-52.26; $P=$ .04) had a significantly higher rate of SSIs. There was a trend suggesting a higher rate of SSIs among patients undergoing clean-contaminated surgery (OR, 3.16; 95\% CI, $0.88-11.41 ; P=.08$ compared to clean cases) and those having received prior radiation (OR, 3.11; 95\% CI, $0.90-10.79 ; P=.07$ ) or chemotherapy (OR, $4.06 ; 95 \% \mathrm{CI}$, 0.93-17.61; $P=.06$; Table IV).

\section{7}

Female

\begin{tabular}{|c|c|c|}
\hline Efficacy of Decols & $\begin{array}{l}\text { 3LE III. } \\
\text { Among Selected Subg }\end{array}$ & \\
\hline Covariate & Odds Ratio $(95 \% \mathrm{Cl})$ & $P$ \\
\hline \multicolumn{3}{|l|}{ Gender } \\
\hline Male & $0.381(0.066-2.193)$ & .28 \\
\hline Female & $0.282(0.047-1.706)$ & .17 \\
\hline \multicolumn{3}{|l|}{ Smoker } \\
\hline Ever & $0.292(0.055-1.553)$ & .15 \\
\hline Never & $0.550(0.068-4.462)$ & .58 \\
\hline \multicolumn{3}{|l|}{ Prior chemotherapy } \\
\hline Yes & $0.333(0.021-5.329)$ & .44 \\
\hline No & $0.355(0.084-1.498)$ & .16 \\
\hline \multicolumn{3}{|l|}{ Prior surgery } \\
\hline Yes & $0.882(0.110-7.058)$ & \\
\hline No & $0.202(0.038-1.082)$ & .06 \\
\hline \multicolumn{3}{|l|}{ Prior XRT } \\
\hline Yes & $0.171(0.015-1.905)$ & \\
\hline No & $0.503(0.110-2.308)$ & \\
\hline \multicolumn{3}{|l|}{ Surgical wound } \\
\hline Clean & $0.316(0.030-3.322)$ & .34 \\
\hline Clean-contaminated & $0.293(0.064-1.348)$ & .12 \\
\hline \multicolumn{3}{|l|}{ Diabetic } \\
\hline Yes & $0.375(0.022-6.348)$ & .5 \\
\hline No & $0.320(0.078-1.319)$ & .1 \\
\hline \multicolumn{3}{|l|}{ ASA classification } \\
\hline 1 & $0.467(0.038-5.734)$ & .55 \\
\hline 3 & $0.771(0.136-4.391)$ & .77 \\
\hline S. aureus carrier & $1.538(0.122-19.461)$ & .74 \\
\hline
\end{tabular}

$\mathrm{Cl}=$ confidence interval; $\mathrm{XRT}=$ external radiation therapy; $\mathrm{ASA}=$ American Society of Anesthesiologists Physical Status classification system. 


\begin{tabular}{lll}
\hline \multicolumn{3}{c}{ TABLE IV. } \\
\hline \multicolumn{1}{c}{ Surgical Site Infection Risk Factors. } & \\
\hline Covariate & \multicolumn{1}{c}{ Odds Ratio (95\% Cl) } & $P$ \\
\hline Gender, male vs. female & $0.661(0.204-2.146)$ & .49 \\
Smoker, yes vs. no & $1.631(0.449-5.917)$ & .46 \\
Prior chemotherapy, yes vs. no & $4.056(0.934-17.611)$ & .06 \\
Prior surgery, yes vs. no & $0.487(0.137-1.736)$ & .27 \\
Prior radiation, yes vs. no & $3.109(0.896-10.785)$ & .07 \\
Surgical wound class & $3.160(0.876-11.408)$ & .08 \\
Diabetic, yes vs. no & $2.566(0.549-12.00)$ & .23 \\
ASA classification & & \\
$\quad 3$ vs. 1 & $4.707(1.068-20.737)$ & .02 \\
$\quad$ 2 vs. 1 & $1.250(0.226-6.902)$ & .45 \\
Operative takeback, yes vs. no & $7.506(1.078-52.261)$ & .04 \\
S. aureus carrier, yes vs. no & $0.641(0.152-2.476)$ & .49 \\
Antibiotic duration, $>24$ vs. & $3.087(0.820-11.616)$ & .10 \\
$\quad<24$ hours & & \\
Patient age, yr & $1.000(0.959-1.042)$ & .99 \\
Case length, min & $1.002(0.99-1.005)$ & .13 \\
Operative EBL & $1.002(1.000-1.004)$ & $.05^{\dagger}$ \\
\hline \hline
\end{tabular}

${ }^{*}$ Compares clean-contaminated to clean cases.

†Statistically significant.

$\mathrm{Cl}=$ confidence interval; ASA = American Society of Anesthesiologists Physical Status classification system; EBL = estimated blood loss.

\section{DISCUSSION}

\section{Topical Antimicrobial Decolonization Before Head and Neck Surgery}

Our data suggest a trend toward a decreased incidence of SSIs with preoperative topical antimicrobial decolonization among head and neck surgical patients; however, there was not a significant difference between the experimental and control groups. Nevertheless, the finding that there were four major infections in the control group, and none in the experimental group, coupled with a low number needed to treat, is interesting. We performed subgroup analyses to determine whether a benefit would be identified among high-risk patients; no specific subgroup demonstrated a significant difference in SSI rate with decolonization, although these analyses were considerably underpowered.

The absence of a statistically significant difference between the control and experimental arms obviates our ability to endorse routine perioperative decolonization in head and neck surgery. That said, given an intriguing trend seen in our study population, this practice might be considered for high-risk groups, despite inconclusive evidence confirming efficacy.

\section{Role of S. aureus in Head and Neck Surgery}

It is estimated that approximately $30 \%$ of people are colonized with $S$. aureus in their anterior nares, pharynx, and various skin sites, which is consistent with our data. ${ }^{17}$ Decolonization of $S$. aureus can be accomplished using a regimen of intranasal mupirocin and bathing with chlorhexidine. ${ }^{18}$ Data regarding the effectiveness of preoperative $S$. aureus decolonization for prevention of SSIs have been mixed. One large study demonstrated that use of mupirocin alone in the preoperative setting did not reduce the risk of SSIs for patients found to have MRSA colonization preoperatively. ${ }^{19}$ Another large study of surgical patients demonstrated that preoperative testing and decolonization (with both mupirocin and chlorhexidine) for MRSA at the time of hospital admission did not change the rate of SSIs due to MRSA. ${ }^{20}$ However, many of the patients in this study were not tested in time to undergo MRSA decolonization prior to surgery, and many patients colonized with MRSA did not receive perioperative antimicrobials effective against MRSA. A more recent, similar study demonstrated that use of mupirocin and chlorhexidine preoperatively in patients colonized with $S$. aureus (both MSSA and MRSA) reduced the rate of SSIs due to $S$. aureus. ${ }^{6}$ All of these studies evaluated for nasal carriage of $S$. aureus only and did not include any patients undergoing head and neck surgery.

There have been three retrospective studies examining the relationship between head and neck surgery and MRSA (but not MSSA) infections. These studies have demonstrated the considerable morbidity and high cost associated with these infections, as well as the efficacy of preoperative decolonization with nasal mupirocin in groups with a high rate of MRSA colonization. Morimoto et al. performed a case-control study of 100 patients, determining that unrestricted use of postoperative antimicrobials and long surgical duration were associated with significantly higher rates of postoperative MRSA infection. ${ }^{21}$ Another case-control study concluded that almost $80 \%$ of head and neck cancer patients in Japan are colonized with MRSA, and that use of perioperative nasal mupirocin was associated with a decrease in SSIs due to MRSA. ${ }^{22}$ In a retrospective analysis in Ireland, $45 \%$ of patients were MRSA-positive postoperatively, of whom more than half required additional surgery, with average hospital stays three times longer than the MRSA-negative cohort. ${ }^{23}$ We did not identify a relationship between MSSA or MRSA carrier status and postoperative infections, and the two patients who developed MRSA SSIs in our study were not colonized preoperatively. There was not a trend suggesting an advantage of decolonization in this population; however, this was not the primary outcome, nor was the study adequately powered for this analysis.

Importantly, most studies examining the efficacy of preoperative decolonization for $S$. aureus have only evaluated for nasal carriage of $S$. aureus. However, it is well known that other body sites are frequently colonized with $S$. aureus. In one study of patients known to have MRSA colonization, only $41 \%$ were found to have colonization of the anterior nares, and 38\% were found to have colonization of the throat. ${ }^{24}$ In $17 \%$ of patients, the throat was the only site of colonization. Another study of hospitalized patients with known MRSA colonization found that performing nasal swab cultures missed $16 \%$ of patients colonized with MRSA. ${ }^{25}$ Other common sites of colonization included the throat (65\% of patients), axillae $(31 \%)$, groin $(38 \%)$, and perineum $(40 \%)$. Sampling of multiple sites greatly increased the sensitivity for detection of MRSA colonization, and our findings are consistent with other published reports in this regard. 


\section{Risk Factors for SSIs}

Specific clinical factors increase the risk for SSIs. This study corroborates findings in general and gynecologic surgery populations, which reported that surgical wound class and ASA classification affect the risk of SSIs. ${ }^{3,26}$ Operative takebacks also increased the risk for SSIs, similar to findings reported among neurosurgical patients. ${ }^{27} \mathrm{~A}$ recent retrospective study identified univariate predictors (prior radiotherapy or chemotherapy, anemia, hypoalbuminemia, mandibulotomy, flap reconstruction, tracheotomy, clean-contaminated cases, blood transfusion, and operation times) and multivariate predictors (oral cavity cancer, prior radiotherapy, tracheotomy, and clean-contaminated cases) of SSIs after head and neck surgery. ${ }^{28}$ Our findings are similar, and variation in results likely reflects differences in patient populations, as well as that our analysis was underpowered for specific risk factor determination. Unlike prior findings among head and neck surgical patients, diabetes did not influence SSI risk, although there were few diabetic patients in this study. ${ }^{29}$ The type of head and neck surgery may also influence the risk for infection; due to the diversity of procedures included in this study, this was not specifically studied. ${ }^{30}$

\section{Strengths and Weaknesses}

This study offers the first prospective, randomized, controlled data on preoperative topical antimicrobial decolonization in head and neck surgery. The patients in the two arms of the study were well matched for demographic and clinical factors, as well as for a number of confounding variables that have been associated with an increased risk for infection in both this study and others. The study's design also facilitated an unbiased characterization of the $S$. aureus carrier rate in this population, both as a descriptor of MSSA and MRSA colonization in multiple anatomic sites, and as a method to assess the efficacy of decolonization within this subgroup.

Despite its careful execution and design, this study has notable limitations. There was no mechanism to determine compliance with the decolonization regimen other than patient self-report. In addition, the intervention was not blinded; however, given the rigid, prospective definition of what constituted an SSI, this is unlikely to have biased the data significantly. Despite our initial power calculation, a lower-than-expected SSI rate limited our ability to detect a significant difference in the primary outcome. One of the major flaws of this study is that subgroup analyses were underpowered, limiting our ability to make salient conclusions regarding the efficacy of the intervention in potentially highrisk populations, including the subgroup of patients colonized with $S$. aureus, who did not have a significantly increased risk for developing an SSI. Our heterogeneous surgical population limits our ability to comment upon the efficacy of decolonization for specific types of head and neck surgery. Similarly, a lack of power obviated the utility of multivariate analyses of potential SSI risk factors. Decolonization is an imperfect process, and we did not quantify or verify the efficacy of decolonization of carriers of MSSA, MRSA, or other potential pathogens. Moreover, more aggressive decolonization protocols involving antimicrobial or antiseptic mouth rinses may have been useful, despite the dearth of data suggesting a benefit of these interventions in prior studies. ${ }^{31,32}$ Finally, there is a risk that preoperative topical antimicrobial decolonization may select for resistant organisms; we did not explicitly study this outcome or its potential consequences. ${ }^{33,34}$

\section{CONCLUSION}

Preoperative topical antimicrobial decolonization did not significantly decrease the incidence of SSIs among head and neck surgical patients. However, given a strong trend indicating the possibility of a benefit and the lack of statistical power among the subgroup analyses, clinicians might consider perioperative topical antimicrobial decolonization for high-risk patient subgroups in the absence of definitive data. Approximately $1 / 3$ of head and neck surgical patients are colonized with $S$. aureus preoperatively, of whom a minority have MRSA; we did not identify a higher risk of infection, nor a trend toward higher efficacy of decolonization in this population. Patients with a higher ASA Physical Status classification system score, those with more operative blood loss, and those who required operative takeback had a higher rate of SSIs, with a trend suggesting a similarly higher rate of SSIs among patients undergoing clean-contaminated surgery and those having received prior radiation or chemotherapy.

\section{BIBLIOGRAPHY}

1. Phan M, Van der Auwera P, Andry G, et al. Antimicrobial prophylaxis for major head and neck surgery in cancer patients: sulbactam-ampicillin versus clindamycin-amikacin. Antimicrob Agents Chemother 1992;36: 2014-2019.

2. Penel N, Fournier C, Lefebvre D, Lefebvre JL. Multivariate analysis of risk factors for wound infection in head and neck squamous cell carcinoma surgery with opening of mucosa. Study of 260 surgical procedures. Oral Oncol 2005;41:294-303.

3. Dellinger EP, Ehrenkranz NJ, Jarvis WR. Surgical site infections. In: Jarvis WR, ed. Bennett and Brachman's Hospital Infections. Philadelphia, PA: Lippincott, Williams, and Wilkins; 2007:583-598.

4. Sutherland R, Boon RJ, Griffin KE, Masters PJ, Slocombe B, White AR. Antibacterial activity of mupirocin (pseudomonic acid), a new antibiotic for topical use. Antimicrob Agents Chemother 1985;27:495-498.

5. Milstone AM, Passaretti CL, Perl TM. Chlorhexidine: expanding the armamentarium for infection control and prevention. Clin Infect Dis 2008;46: 274-281.

6. Bode LG, Kluytmans JA, Wertheim HF, et al. Preventing surgical-site infections in nasal carriers of Staphylococcus aureus. $N$ Engl $J \mathrm{Med}$ 2010;362:9-17.

7. van Rijen M, Bonten M, Wenzel R, Kluytmans J. Mupirocin ointment for preventing Staphylococcus aureus infections in nasal carriers. Cochrane Database Syst Rev 2008(4):CD006216.

8. van Rijen MM, Bonten M, Wenzel RP, Kluytmans JA. Intranasal mupirocin for reduction of Staphylococcus aureus infections in surgical patients with nasal carriage: a systematic review. J Antimicrob Chemother 2008; 61:254-261.

9. Webster J, Osborne S. Preoperative bathing or showering with skin antiseptics to prevent surgical site infection. Cochrane Database Syst Rev 2007(2):CD004985

10. Hebert C, Robicsek A. Decolonization therapy in infection control. Curr Opin Infect Dis 2010;23:340-345.

11. Mangram AJ, Horan TC, Pearson ML, Silver LC, Jarvis WR. Guideline for prevention of surgical site infection, 1999. Hospital Infection Control Practices Advisory Committee. Infect Control Hosp Epidemiol 1999;20: 250-278; quiz 279-280.

12. Dellinger EP, Gross PA, Barrett TL, et al. Quality standard for antimicrobial prophylaxis in surgical procedures. The Infectious Diseases Society of America. Infect Control Hosp Epidemiol 1994;15:182-188. 
13. Horan TC, Andrus M, Dudeck MA. CDC/NHSN surveillance definition of health care-associated infection and criteria for specific types of infections in the acute care setting. Am J Infect Control 2008;36:309-332.

14. Simmons RL. Wound infection: a review of diagnosis and treatment. Infect Control 1982;3:44-51.

15. Dripps RD, Lamont A, Eckenhoff JE. The role of anesthesia in surgical mortality. JAMA 1961;178:261-266.

16. ASA Physical Status Classification System. Available at: http://www.asahq org/clinical/physicalstatus.htm Accessed June 3, 2011.

17. Que Y, Moreillon P. Staphylococcus aureus. In: Mandell GL, Bennett JE, Dolin R, eds. Mandell, Bennett, and Dolin's Principles and Practice of Infectious Diseases. 7th ed. Philadelphia, PA: Elsevier; 2009.

18. Buehlmann M, Frei R, Fenner L, Dangel M, Fluckiger U, Widmer AF. Highly effective regimen for decolonization of methicillin-resistant Staphylococcus aureus carriers. Infect Control Hosp Epidemiol 2008;29. 510-516.

19. Perl TM, Cullen JJ, Wenzel RP, et al. Intranasal mupirocin to prevent postoperative Staphylococcus aureus infections. N Engl J Med 2002;346: 1871-1877.

20. Harbarth S, Fankhauser C, Schrenzel J, et al. Universal screening for methicillin-resistant Staphylococcus aureus at hospital admission and nosocomial infection in surgical patients. JAMA 2008;299:1149-1157.

21. Morimoto Y, Sugiura T, Tatebayashi S, Kirita T. Reduction in incidence of methicillin-resistant Staphylococcus aureus (MRSA) after radical surgery for head and neck cancer. Spec Care Dentist 2006;26:209-213.

22. Miyake M, Ohbayashi Y, Iwasaki A, Ogawa T, Nagahata S. Risk factors for methicillin-resistant Staphylococcus aureus (MRSA) and use of a nasal mupirocin ointment in oral cancer inpatients. J Oral Maxillofac Surg 2007;65:2159-2163.

23. Watters K, O'Dwyer TP, Rowley H. Cost and morbidity of MRSA in head and neck cancer patients: what are the consequences? J Laryngol Otol 2004;118:694- 699
24. Ringberg H, Cathrine Petersson A, Walder M, Hugo Johansson PJ. The throat: an important site for MRSA colonization. Scand $J$ Infect Dis $2006 ; 38: 888-893$.

25. Lautenbach E, Nachamkin I, Hu B, et al. Surveillance cultures for detection of methicillin-resistant Staphylococcus aureus: diagnostic yield of anatomic sites and comparison of provider- and patient-collected samples. Infect Control Hosp Epidemiol 2009;30:380-382.

26. Garibaldi RA, Cushing D, Lerer T. Predictors of intraoperative-acquired surgical wound infections. J Hosp Infect 1991;18(suppl A):289-298.

27. Korinek AM, Golmard JL, Elcheick A, et al. Risk factors for neurosurgical site infections after craniotomy: a critical reappraisal of antibiotic prophylaxis on 4,578 patients. Br J Neurosurg 2005;19:155-162.

28. Lee DH, Kim SY, Nam SY, Choi SH, Choi JW, Roh JL Risk factors of surgical site infection in patients undergoing major oncological surgery for head and neck cancer. Oral Oncol 2011;47:528-531.

29. Rodrigo JP, Alvarez JC, Gomez JR, Suarez C, Fernandez JA, Martinez JA Comparison of three prophylactic antibiotic regimens in clean-contaminated head and neck surgery. Head Neck 1997;19:188-193.

30. Skitarelic N, Morovic M, Manestar D. Antibiotic prophylaxis in clean-contaminated head and neck oncological surgery. J Craniomaxillofac Surg 2007;35:15-20.

31. Redleaf MI, Bauer CA. Topical antiseptic mouthwash in oncological surgery of the oral cavity and oropharynx. J Laryngol Otol 1994;108:973-979.

32. Simons JP, Johnson JT, Yu VL, et al. The role of topical antibiotic prophylaxis in patients undergoing contaminated head and neck surgery with flap reconstruction. Laryngoscope 2001;111:329-335.

33. Fawley WN, Parnell P, Hall J, Wilcox MH. Surveillance for mupirocin resistance following introduction of routine peri-operative prophylaxis with nasal mupirocin. J Hosp Infect 2006;62:327-332

34. Lee AS, Macedo-Vinas M, Francois P, et al. Impact of combined low-level mupirocin and genotypic chlorhexidine resistance on persistent methicillin-resistant Staphylococcus aureus carriage after decolonization therapy: a case-control study. Clin Infect Dis 2011;52:1422-1430. 